\title{
Considerations for the Design of Pre-exposure Prophylaxis (PrEP) Interventions for Women: Lessons Learned from the Implementation of a Novel PrEP Intervention
}

\author{
Suzan M. Walters ${ }^{1}$ (D) Joey Platt ${ }^{2} \cdot$ Amarachi Anakaraonye $^{2}$. Sarit A. Golub ${ }^{3}$. Chinazo O. Cunningham ${ }^{2}$. \\ Brianna L. Norton ${ }^{2}$. Jae M. Sevelius ${ }^{4}$. Oni J. Blackstock ${ }^{2}$
}

Accepted: 11 June 2021 / Published online: 17 June 2021

(c) The Author(s), under exclusive licence to Springer Science+Business Media, LLC, part of Springer Nature 2021

\begin{abstract}
Pre-exposure prophylaxis (PrEP) uptake among women in the United States has been low. To increase uptake, we developed a peer outreach and navigation PrEP intervention. Semi-structured qualitative interviews with 32 cisgender women and 3 transgender women were conducted to assess the intervention. We used a thematic approach to identify barriers to, and facilitators of the intervention. Facilitators included interest in PrEP, offer of health and social services, the intervention's women-focused approach, and peer outreach and navigation. Barriers were perceived HIV risk, concerns about medication side effects or interactions, housing insecurity and travel, co-occurring health-related conditions, and caregiving responsibilities. We recommend that future interventions consider packaging PrEP in local community settings, such as syringe exchange programs; include services such as food and housing assistance; use peers to recruit and educate women; integrate a culturally appropriate women's focus; and consider providing same-day PrEP.
\end{abstract}

Keywords Cisgender women $\cdot$ Transgender women $\cdot$ Pre-exposure prophylaxis $\cdot$ HIV $\cdot$ Syringe service program $\cdot$ Drug use $\cdot$ Exchange sex

\section{Introduction}

Research indicates that exchange sex (i.e., sex in exchange for money, drugs, or other goods or services) increases HIV risk among women [1-4]. Among women in the United States (U.S.) who engage in exchange sex, HIV prevalence among cisgender women is estimated to be $23 \%$ [5] and as high as $27 \%$ among transgender women [6]. Compared with white women, women of color, particularly African

Suzan M. Walters

smk556@nyu.edu

1 School of Global Public Health, New York University, New York, NY, USA

2 Division of General Internal Medicine, Department of Medicine, Montefiore Medical Center/Albert Einstein College of Medicine, 3300 Kossuth Avenue, Bronx, NY 10467, USA

3 Department of Psychology, Hunter College, City University of New York, New York, NY, USA

4 Department of Medicine, University of California San Francisco, San Francisco, CA, USA
American/Black and Latina women, have higher HIV prevalence [5]. In addition to sexual contact, substance use, including injection drug use, is an additional HIV risk factor for cisgender and transgender women [7]. Injection drug use accounts for 5.6\% of new HIV diagnoses in the U.S., and there are indications that rates of new HIV diagnoses may be increasing among persons who inject drugs, as there have been several recent outbreaks of HIV in the US attributed to injection drug use [8-10].

Difficulties experienced by women who use drugs include negotiating sterile injecting equipment with a partner (i.e., receptive syringe sharing) [26] and negotiating condom use during sex with partners [7] due to power relations and social conditions [11]. Many women who engage in streetbased exchange sex and/or injection drug use experience poverty, gender-based power dynamics, social isolation, and violence which serve to undermine safety negotiations [12-17]. Barriers to health care, including social stigma, fear of arrest, and fear of discrimination by health care providers, further elevate risk of HIV acquisition by increasing avoidance and limiting access to comprehensive HIV prevention services $[18,19]$. 
Pre-exposure prophylaxis (PrEP) is effective in reducing the risk of HIV acquisition for women at high risk for HIV infection when taken regularly [20-24]. As a user-controlled HIV prevention method, PrEP may be particularly important for women who engage in exchange sex and/or use drugs, especially those who inject drugs who may be at risk for HIV and struggle in negotiating condoms or drug use equipment $[25,26]$. However, despite PrEP's potential benefit, PrEP uptake in the U.S. have been persistently low among women [27-31].

One of the main barriers to PrEP for women is lack of PrEP awareness [7, 25, 32, 33]. Without being aware that PrEP exists, women are unlikely to be able to acquire the medication and take it. Other barriers include perceived low risk of HIV [34] and misinformation about PrEP eligibility and appropriateness [35]. Importantly, women who perceive their risk as low have reported behaviors, such as condomless sex and sharing injection equipment, indicating they may be at greater risk than perceived [34, 36], thus highlighting the importance of HIV prevention education including information about PrEP. Concerns about side effects and cost of PrEP [35, 37-39], reduced efficacy if not taken daily [31], PrEP-related stigma [39-41], and medical mistrust [37], including mistrust due to transphobia experienced by women of trans experience [42], have also been identified in previous studies. Along with barriers some studies have identified facilitators to PrEP uptake, such as learning about PrEP from a trusted source [43] and having a welcoming, client-centered, culturally appropriate environment that is stigma free allowing for positive interactions with PrEP prescribers [35, 43, 44].

Women have also indicated a preference for acquiring PrEP information and PrEP itself through peer educators and community-based organizations $[45,46]$. Peer outreach and navigation has been successful in the context of HIV medical care to engage with and connect individuals (often from marginalized and underserved populations) to health care and social services [47-49]. Peer workers have also been associated with improved outcomes for people in mental health [50, 51] and substance use care [52] as well as harm reduction [53]. Peers help women navigate healthcare systems, overcome system barriers, advocate for women who need help, and are able to restore trust in the healthcare system [54]. A successful peer is someone who has shared experiences with the target population, is sociable, reliable, respectful, open-minded, and good at listening [54]. To our knowledge there have been no published peer-based PrEP interventions focused on increasing PrEP uptake among cisgender and transgender women and persons with other transfeminine identities.

We developed, and pilot tested a peer outreach and navigation intervention called PrEP-UP to increase access to PrEP for women at high risk for HIV. PrEP-UP was delivered in collaboration with an established communitybased organization serving women who engage in drugs use and/or exchange sex. The intervention and outcomes from the pilot study are described elsewhere [55]. It is important to note is that no women started PrEP. This analysis focuses on follow-up qualitative interviews conducted with intervention participants to understand their experiences with PrEP-UP including what aspects of the intervention were well-received and what modifications would help to increase engagement in the intervention, and, ultimately, PrEP uptake. Thus, this paper focuses on why women did not ultimately start PrEP and how future interventions might be able to better engage women.

\section{Methods}

\section{Setting and Design}

The peer outreach and navigation intervention was delivered at a community-based organization (CBO) located in two regions of New York City with high HIV prevalence: East Harlem and the Bronx, NY [56]. The CBO had nine mobile syringe exchange sites as well as two fixed locations, which included a drop-in center specifically for women involved in sex work. The CBO provided harm reduction services and tools, such as condoms, pipes for smoking drugs such as crack cocaine and/or methamphetamines, opioid overdose prevention services, and syringe exchange; HIV and hepatitis $\mathrm{C}$ testing; case management; mental health care; referrals to substance use treatment programs; housing placement assistance, and benefits assistance. Most services were delivered by peer outreach workers (i.e., peers), which helped facilitate the integration of peers for this study.

Guided by the Information-Motivation-Behavioral Skills model of HIV preventive behavior [57], we designed PrEP-UP, which focused on (1) PrEP education, (2) PrEP counseling, and (3) navigation to PrEP care. PrEP-UP was integrated into the CBOs existing peer-facilitated service delivery model. The intervention was delivered by one of two peers (one transgender one cisgender woman) who shared a history of drug use and/or sex work with the participants. The training that the peers received involved content and messaging tailored for cisgender and transgender women which included framing PrEP as a tool to allow women to take control of their sexual health and addressing concerns about interactions with gender-affirming hormone therapy. Peers would approach women who used the CBOs services to offer PrEP UP's services. Education and counseling were delivered in the initial encounter, which lasted approximately 10-15 min. The education component consisted of providing the basic facts about PrEP (i.e., what PrEP is, how it works, what 
PrEP does not do, side effects, and what is involved in taking PrEP). The counseling component utilized motivational interviewing techniques to address attitudes about and behavioral intentions towards initiating and engaging in PrEP care and taking PrEP. PrEP information was tailored for women (i.e., PrEP as a tool to empower women to take control of their sexual health), which included information about potential interactions between PrEP and gender-affirming hormone therapy for transgender women [39, 42, 58]. After education and counseling, participants were asked if they were interested in learning more about PrEP. If they expressed interest in PrEP, participants were offered a PrEP care appointment at a nearby clinic in the South Bronx where physicians specialized in PrEP care. The clinic was accessible via public transportation and offered primary and subspecialty care; buprenorphine treatment; HIV and Hepatitis C Virus treatment; genderaffirming care and services, and referrals to outpatient and inpatient substance use treatment. In addition to the peer navigators in this study, the clinic had a dedicated PrEP patient navigator who called patients with appointment reminders; followed up with patients after missed visits; linked patients to relevant social services in the community and assisted with insurance coverage issues. At that time, peers also addressed additional health concerns that women expressed (e.g., primary care, obstetrics/gynecology) and social service needs (e.g., housing, mental health services, substance use treatment, case management) by providing referrals. Throughout the PrEP-UP intervention peers assisted with appointment scheduling, provided appointment reminders, transportation to appointments, and accompaniment to PrEP care appointments. More detailed information on the PrEP-UP intervention has previously been published [55]. This study reports the findings from semi-structured qualitative interviews that were conducted with women to assess their experiences with the PrEP-UP intervention. The study was approved by Albert Einstein College of Medicine's Institutional Review Board Institutional Review Board (IRB).

\section{Participants}

Eligibility requirements included being self-identified as a woman (including trans women and other trans feminine identities), 18 years or older, proficient in English, and receiving services from the partner $\mathrm{CBO}$. We kept the eligibility criteria broad given that women using these services were likely to be at high risk of HIV acquisition due to related activities (e.g., condomless sex, sex work, drug use) and because we wanted to minimize the likelihood of potential participants providing socially desirable responses to meet study eligibility. Women who reported being HIV-positive in the baseline survey were offered the CBOs usual services which included linkage and navigation to any needed healthcare or social services, including HIV care.

\section{Data Collection}

Qualitative interviews took place at the partner CBOs mobile syringe exchange sites and fixed street-side locations. Quantitative data, which included socio-demographic information, were collected using surveys and peer activity logs. Between four and twelve weeks after enrollment (December 2017-May 2018), we conducted semi-structured qualitative interviews with participants. The qualitative interviews elicited barriers to and facilitators of engagement in the PrEP-UP intervention as well as participants' impressions of PrEP-UP and reasons for participation. We asked participants why they participated in PrEP-UP, as well as pointed questions about their interest, or lack of, in PrEP. If women declined the offer of referral for PrEP or did not attend an initial PrEP care appointment, we explored why, depending on where each woman fell on the continuum. After asking those questions, we asked "What could we have done to increase your interest in PrEP?" We asked questions about their experience with PrEP-UP. For example, we asked, "What did you think about interactions with your peer?" and "What changes would you suggest be made to the program?" All qualitative interviews were conducted either in-person or over the phone using a mobile recording application. Phone interviews were available to participants who were unable to meet the study staff in-person. Interviews were professionally transcribed. Participants received a $\$ 25$ gift card for their participation in the follow-up semi-structured qualitative interview along with a roundtrip MetroCard (value \$5) for those that attended the follow-up interview in-person. We offered financial incentives because past research has shown increased participation when incentives were offered $[59,60]$. In addition, incentives can build trust with participants by demonstrating a respect for the participants time and effort [61].

\section{Analysis}

Interviews were audio-recorded and professionally transcribed. After transcription they were read for accuracy and uploaded to Dedoose software for data management. At that time three coders independently coded 10 interviews using a deductive coding approach [62]. Coders first familiarizing themselves with the data, then generating initial codes, and finally grouped codes into themes [63]. This approach was taken to achieve saturation of major themes and to identify any emergent themes. After coding the first ten transcripts separately, the coding team reconciled codes through 
discussion that aligned and diverged across all the interviews to create a consolidated and finalized code book. The consolidated code book, which included the code, description of the code and when to use the code [64], was used to recode the initial ten transcripts and the remaining transcripts. Discrepancies in coding were resolved through consensus $[62,65,66]$.

\section{Results}

Of the 35 HIV-negative women we interviewed, 18 (51\%) had previously heard about PrEP. After receiving the intervention, 24 (69\%) expressed interest in PrEP, 16 (46\%) accepted an offer to schedule a PrEP care appointment, 12 (34\%) received a scheduled appointment, and none attended a PrEP appointment. Three (9\%) were transgender women and $32(91 \%)$ were cisgender women (Table 1). Almost half $(46 \%)$ were age 50 or older. The racial/ethnic breakdown of the women was $46 \%$ Latina and $46 \%$ non-Latina Black. Over half (63\%) identified as bisexual, lesbian, gay or other. Despite $34 \%$ reporting a high school diploma or GED and $31 \%$ reporting some college or more, $40 \%$ were housing insecure (i.e., not having their own apartment or house). Many women reported behaviors that might put them at risk for HIV, such as condomless vaginal sex (40\%), condomless anal sex (14\%), exchange sex (23\%), and substance use $(71 \%)$. We found that $11 \%$ of women reported injecting drugs in the last 30 days and $76 \%$ of women reported having a main sex partner who injected drugs.

\section{Facilitators to PrEP-UP Intervention Engagement}

Several aspects of the intervention were well-received by participants. The themes that emerged were (1) interest in PrEP, (2) offering health and social services other than PrEP care, (3) the women-focused approach, and (4) peer outreach and navigation.

\section{PrEP Interest}

Many women we encountered were either unaware of PrEP or heard about PrEP but did not have a full understanding of what PrEP was. For example, Aliyah, a 47-year-old African American cisgender women, told us "I did hear of PrEP, but I had never learned anything about it. So, I wanted to learn exactly what it was." Like Aliyah, women were motivated to learn more about PrEP because they understood PrEP as a potential resource that could benefit their health.

For example, Elois, a 52-year-old cisgender African American woman, who did not know what PrEP was prior to participating in the study, expressed her desire to gain
Table 1 Participant Characteristics $(\mathrm{n}=35)$

\begin{tabular}{|c|c|}
\hline Characteristic & $\mathrm{n}(\%)$ \\
\hline \multicolumn{2}{|l|}{ Sociodemographic } \\
\hline \multicolumn{2}{|l|}{ Gender } \\
\hline Cisgender woman & $32(91)$ \\
\hline Transgender woman & $3(9)$ \\
\hline \multicolumn{2}{|l|}{ Age } \\
\hline $25-35$ & $8(23)$ \\
\hline $36-50$ & $11(31)$ \\
\hline $50+$ & $16(46)$ \\
\hline \multicolumn{2}{|l|}{ Race/ethnicity } \\
\hline Hispanic/Latina & $16(46)$ \\
\hline Non-Hispanic Black & $16(46)$ \\
\hline Non-Hispanic white & $3(9)$ \\
\hline \multicolumn{2}{|l|}{ Sexual orientation } \\
\hline Heterosexual & $13(37)$ \\
\hline Bisexual, lesbian, gay, or other & $22(63)$ \\
\hline \multicolumn{2}{|l|}{ Education } \\
\hline Some high school & $12(34)$ \\
\hline High school diploma or GED & $12(34)$ \\
\hline Some college or more & $11(31)$ \\
\hline Housing insecure & $14(40)$ \\
\hline \multicolumn{2}{|l|}{ Sexual behaviors } \\
\hline Main partner has other sex partner(s) ${ }^{\mathrm{a}}$ & $11(52)$ \\
\hline Condomless vaginal sex & $14(40)$ \\
\hline Condomless anal sex & $5(14)$ \\
\hline Sex with exchange partner & $8(23)$ \\
\hline \multicolumn{2}{|l|}{ Substance and drug use } \\
\hline Any substance use (past 30 days) & $25(71)$ \\
\hline Injected drugs (past 30 days) & $4(11)$ \\
\hline Main partner injects drugs ${ }^{\mathrm{a}}$ & $16(76)$ \\
\hline \multicolumn{2}{|l|}{ Psychosocial conditions } \\
\hline Depression & $16(46)$ \\
\hline Anxiety & $20(57)$ \\
\hline Intimate partner violence & $8(23)$ \\
\hline \multicolumn{2}{|l|}{ PrEP } \\
\hline Heard of PrEP & $18(51)$ \\
\hline PrEP interest & $24(69)$ \\
\hline Previously prescribed PrEP & $2(11)$ \\
\hline
\end{tabular}

${ }^{a}$ Indicates missing data due to skip patterns in the quantitative survey

more knowledge about PrEP in order to protect herself. She described how being sexually active influenced her decision to enroll in the study. Elois said,

Because I needed to know those things, especially when she was explaining to me about the HIV testing. She said, "Have you ever heard of PrEP?" She had told me, and I said, "No, what is that?" She started telling me. I wanted to find out what's going 
on. And I'm sexually active. I'm at the age too now there's a lot of things I don't know. I definitely liked that women helping women. That's what I need.

Another participant, Barbara, a 52-year-old cisgender Latina woman, felt that the PrEP-UP intervention was something she needed as a woman who engaged in sex work and injection drug use. She expressed that the study could be helpful for her and for other women she knew. When we asked about why she participated in the study, she commented about her desire to not only educate herself, but also other women, saying:

Because it's something that I needed for myself. It's something that I want to make sure that I'm good about. And I could talk to other women that are going through the same thing I'm going through. And not even other women. I know a lot of girls out there that still use, I know a lot of girls that still, they be out there, selling their body. So, I try to talk to them a little bit about it.

Whether participants were interfacing with our CBOs mobile syringe unit or drop-in centers, many expressed that they enrolled in the study because they were curious about PrEP.

Offer of health and social services Although many of the participants initially participated in the study to receive the financial incentive, the incentive often served as a gateway to obtain other needed health or social services and/or obtain sexual health education for themselves, or to share with other women in their lives, and women noted these services as a draw for them. For example, Cari, a 50-year-old, Black, cisgender woman who was currently living in a shelter commented on how her participation in the study served as a gateway to those resources:

I participated in it [PrEP-UP] because I heard from one of my roommates that she learned a lot from it, and she don't know what she's gonna do as far as her doctor. She has a different doctor than I did. So, when she told me, I said too, "I wonder if these people can help me get that OBGYN doctor or diabetic stuff." Because I have diabetes it's under control, but I still need to see a doctor that only deals with diabetes. And I'm yet to find one because my doctor hasn't found any for me. So, all of those things ... So this is why I said what I said that you guys most likely can help me get those help. So yeah, I'm happy with it. And for me it's good I have no problem.

When we asked Cari if she followed up on the services that we referred her to she said "No, I haven't been active ... Because I don't remember much...All I know is that
I needed that OBGYN and the colonoscopy." After the interview we had the peer follow up again about the referrals, but to our knowledge Cari did not use any of them.

Like Cari, many women in our study were living in unstable housing and reported food and housing insecurity. As such, a lot of the additional services requested related to these basic needs. For example, Donna, a 43-year-old cisgender Black and Latina woman requested help with food and housing. When we asked her about the PrEP-UP intervention she told us how the additional services piqued her interest. When Donna came to the CBOs site, she was pleasantly surprised when she "realized there's more than just one program here. It's a multi-service building."

However, when we gave Donna referrals that required follow up outside of the CBO for housing and food, she was not able to access them. She told us that she had started a new job and was "overwhelmed".

Since many women had co-occurring conditions and competing priorities, which included housing and food insecurity, interest in additional services was common. In addition to providing referrals to such services, women reported liking PrEP-UP because it was focused on women.

Women-focused approach The study was framed as "women helping women," which was an appeal for participants. In fact, the exclusivity of only women being able to participate (and that the peers were women) was a factor that led some women to participate in the study. As Brenda, a cisgender woman said, "I like the idea of Women Helping Women."

Other women noted the importance of a woman-focused program. Florencia a 50-year-old, Latina, cisgender woman who participated in PrEP-UP was interested in participating because she was yearning for comradery with other women. After talking with Florencia, we learned that she was not interested in PrEP care specifically, she was interested in other social services being offered. She expressed a desire to take classes in order to learn new skills and share her acquired knowledge with other women. She commented on the significance of being in community comprised of other women, saying:

When they said $\$ 40$, of course, it did attract me. But when they said women and women, that's another thing. If they would have said $\$ 40$ men and women, I would have said no. I don't like to be involved in coed. No coed for me, just strictly women.

Participating in a women-focused intervention that included access to gender affirming care was highly valued by transgender women participants. Although only three transgender women were interviewed, two out of three noted the importance for transgender women to be referred to by 
their correct pronoun and treated as a woman. For example, when asked what she liked about the program, Natasha, a 57-year-old Black transgender woman said,

I like everything that-Everybody's so welcoming. They don't judge. They call you she, they call you her despite what you look like. They respect you, and treat you like a human.

Natasha told us that other people she encountered did not treat her this way and that she sometimes would refrain from services because they were not gender affirming. Specifically, she said people might talk nicely to her "but their eyes say something different" when she accessed services not related to the PrEP-UP intervention. Natasha was grateful for the PrEP-UP staff who treated her like any other woman.

Peer navigation Participants expressed overall favorable experiences with the PrEP-UP intervention and found that the peers' involvement had a significant and positive impact. The peers were viewed as personable, knowledgeable and a key aspect of the PrEP-UP intervention. Donna, a 43-yearold cisgender Black and Latina participant commented on how her peer's engaging personality enhanced her comfort as a participant in the study:

She's very informative. She knows a lot of stuff, preventive care, and she knows how to speak to people. It's like somebody where you come from in your own neighborhood. It's a familiar face. You probably listen more. It catches the attention of the person listening.

While peers provided the PrEP education and counseling, participants often discussed other aspects of their lives. Participants noted how the peers facilitated a safe space whereby they could share their stories, particularly those related to trauma. Natasha, a 57-year-old Black transgender woman, commented on the emotional significance of having someone listen to her, saying:

I learned to love myself a little more. [Peer], I cried with them. I told them my father raped me. To be so supportive like that showed me a lot. I never get that. I'm usually the one doing that. I'm usually the one taking care of, and it's really hard for me.

Peer navigation was a critical part of the PrEP-UP intervention that motivated women to participate. Peers familiarized participants with the study and helped them understand the processes of being in a research study, they informed women about PrEP and other services, and they followed up with women to remind them about their appointments as well as offered to attend appointments with them. Following up was not always an easy task because not all women had cell phones. Given that peers had lived experience and were part of the community they were often able to use their insider knowledge and social networks to find women. The fact that peers had shared life experiences with the participants of this study not only benefited retention for the intervention in that we were able to locate hard to reach participants, but it also made participants feel comfortable and respected.

\section{Barriers to PrEP-UP Intervention Engagement}

Our qualitative analysis identified a number of themes related to barriers to engaging in the PrEP-UP intervention. The barriers identified were (1) perceived HIV risk, (2) concerns about medication side effects or interactions, (3) housing insecurity and travel, (4) co-occurring health-related conditions and appointments, and (5) caregiving responsibilities. We chose not to focus on caregiving as that has already been established in the literature [67-70].

\section{Perceived HIV Risk}

Regarding the proximal stages of the PrEP-UP intervention (i.e., PrEP interest and acceptance of a PrEP appointment), the main barrier identified was lack of perceived HIV risk. For the most part, the higher the perceived HIV risk, the more likely participants were to accept a PrEP appointment offer. Monogamy, or not being currently sexually active, were common reasons that women felt they were not at risk for HIV, and, therefore, they felt they did not need PrEP. For example, when asked about PrEP, Patrice, a Black, cisgender woman, age 56, said, "I'm not sexually active, so I didn't feel the need to do that right now." Patrice originally expressed interest in PrEP but did not accept a PrEP appointment because of her perceived low risk for HIV.

Importantly, women often spoke about their HIV risk in solely sexual terms, like Patrice above. Another participant, Jada, a 53-year-old Black, cisgender woman, told us that she was not interested in PrEP.

Because I'm back to being monogamous. I am not sexually active. That's why I did not really need that because I am not sexually active. I can save that for somebody who needs it.

Overall, women who felt that their risk for HIV was low were less interested in PrEP. Along with lack of perceived HIV risk, participants discussed concerns with how their bodies might react to PrEP. Below we discuss how some women had concerns about medication side effects.

\section{Concerns about Medication Side Effects or Interactions}

Even though part of our intervention was to discuss potential side effects to PrEP and inform participants about the 
processes of taking PrEP, some women were concerned about medication side effects. For example, some women expressed concerned about interactions with other medications that they were taking for chronic illnesses. Aliya, a 47-year-old Black, cisgender woman felt she might benefit from PrEP, and was interested in PrEP, but did not accept a PrEP appointment offer said:

I didn't want to mix up-I'm taking a lot of medications. I was asking what the aftereffects from that medication are, and she actually couldn't tell me what the aftereffects were. I didn't want to mix all that medication together. I told her I was interested in that because I'm on the inside and my old man is outside, and I don't know what the hell he's doing. I want to take that pill just in case he is acting stupid.

While the fear of a "monogamous" partner not actually being monogamous was a theme that emerged from the data, Aliya and others were not willing to pursue PrEP, even though she was concerned about her partner's behaviors. Another participant, Frances, a Black, cisgender woman, age 40, said something similar when asked why she did not accept a PrEP appointment offer. Frances described being concerned about medication interactions, but also worrying about how safe PrEP was to take while pregnant. Frances said,

Because I was telling them that I need to consult with my doctor regarding what medications did, and I'm more about the benefits and the risks of the medication.... I thought I was pregnant. Because I'm on, already on, like certain medications that I would want to make sure that it wouldn't interfere with the rest of the medications I'm taking.

After Frances told us how she felt and described her concerns, the interviewer asked if Frances would like to talk to one of the primary care prescribers for this study. Frances declined, saying:

I would like seeing my own doctor. I'm not too particular about seeing other doctors right now. I have my own private doctor. He takes care of most of my medical. Put it like this, I'm on psychiatric medications. A lot of other things scare me. I don't jump right into it.

Concerns about PrEP side effects (including medication interactions), and the desire to have one prescribing physician who handled all of their medical needs kept some women from accepting a PrEP appointment, and from ultimately taking PrEP. Given concerns about interactions with other medications, and the fact that other physicians had prescribed those medications, women seemed to display more comfort in working with their regular doctor, as Frances explained above.

\section{Housing Insecurity and Travel}

Prioritizing stable permanent housing was a recurring theme which seemed to limit women's interest in PrEP, as well as their ability to attend a PrEP care appointment. For example, when peers talked about PrEP with women, participants would often redirect the conversation to housing because they wanted, and needed, housing referrals more than they felt they needed PrEP referrals.

These participants were trying to secure housing (e.g., a homeless shelter, single-room occupancy, etc.), and/or dealing with the residual mental, physical, and/or emotional effects of being homeless. Brenda, A 64-year-old cisgender, Black woman who was living in a nearby homeless shelter expressed that she was not interested in learning more about PrEP because identifying more stable housing was her primary concern. She said,

Right now, I know you don't want to hear all this, but right now, my focus is on getting me a place to stay, to live. That's my alternative main goal is trying to get out of the shelter system and trying to find me someplace.

While some participants' desire to secure more stable housing took precedence over learning about PrEP, for other housing insecure participants, their housing status made attending their PrEP appointments challenging. In fact, all of the participants who reported housing insecurity and expressed interest in receiving a PrEP appointment were unable to attend their scheduled PrEP appointment. April, a 59-year-old cisgender, Black woman was interested in PrEP and scheduled a PrEP appointment; however, she was unable to attend her appointments because she was sleeping on the streets at the time of her scheduled appointment. Unable to secure housing in a homeless shelter at night, the participant slept during the daytime. When she was asked to elaborate on how her lack of secure housing impacted her appointment attendance, she said the following:

My problem is being where I'm supposed to be at the right time, and that has a lot to do with being late with some things. In other words, like if I knew I have appointment, I'll be somewhere maybe getting some sleep, because I didn't sleep at all the night before, because I have nowhere to sleep.

While some women spoke about housing insecurity being an obstacle to traveling to appoints other women talked explicitly about difficulties traveling to appointments in relation to distance and time constraints. For example, Cari, a Black cisgender woman, age 50 said, 
It's way too far the distance for me...for me to get there it would take me two buses and a bit of walking. So, I told her that I can't do that because it's too much on my body with my legs to travel that far.

Donna, a 43-year-old Black and Latina, cisgender woman had an appointment scheduled, but did not attend her appointment. She said,

It's just I prefer not [location of the study]. That's too far for me. I need something local. I'm not going to go far.

When we asked if Donna would go to a PrEP appointment near her, she said she would. Donna, like many others, had conflicting needs, such as housing and food insecurity. We had referred her to services for both, but at the time of the follow up qualitative interview she had not accessed those services. She told us that she had started a new job and that she felt "overwhelmed".

Feelings of being overwhelmed were common, Patricia a 55-year-old white cisgender women said,

I'm overwhelmed half the time with everything that's going on. It seems like it keeps getting shoveled on my shoulders and nothing's getting taken off. I'm a mess right now.

Feelings of being overwhelmed often reflected larger inequalities such as housing insecurity, which also impacted ability to travel to PrEP appointments. In the next section we discuss how co-existing health conditions impeded participants ability to attend PrEP appointments.

\section{Co-occurring Health-Related Conditions and Appointments}

Many participants reported being unable to engage in PrEP care because they had to address other issues related to their physical, behavioral, and/or psychological health, and they did not have additional time to take on appointments for PrEP. Participants' primary health concerns mainly related to chronic medical and psychiatric conditions, as well as substance use. Related to mental health concerns, participants often recounted experiences of childhood and/or adolescent trauma and residual psychological distress into adulthood including in the context of their interpersonal relationships. Participants expressed that other appointments for health-related issues often prevented them from attending scheduled PrEP care appointments or took priority over PrEP appointments.

For example, Jamie, a 37-year-old cisgender Latina woman who had sustained injuries in a prior car accident, was unable to attend her scheduled PrEP appointment because of the many appointments she had related to her injuries, as well as with challenges related to caregiving responsibilities.

Oh, it's just so much going on right now, like I don't have babysitters and all these appointments. I have physical therapy. We were in a car accident, so they just got me going to the doctors. It's so many doctors' appointments I couldn't keep up.

Another participant, Linda, a 51-year-old cisgender Latina woman, was unable to attend her PrEP appointment due to multiple co-existing health conditions. In addition to having asthma, the participant experienced memory loss due to previous strokes that impacted her ability to remember when she had appointments. Due to her memory loss, she specifically requested that her peer provide her multiple appointment reminders prior to and the day of her appointment. Although Linda's peer provided her the reminders she requested, she ultimately did not attend her PrEP appointment because she forgot about it:

I forget things...I have like a memory loss, I got two minor strokes and I have a memory loss so I was telling [peer] that if she can help me out... I was in the hospital, I caught an asthma attack, I was in, I went to that mobile on [location] and when I got there, they took me to Urgent Care because I couldn't breathe, right. And then, the next day I ended up over here on [location] at another, at like a hospital, like a clinic or something. And then they also, they give me a treatment for my asthma, right. And then that day I had an appointment for welfare, for food stamps and I missed it. So, those are the things that happen to me.

Linda articulates the complexities of her life and how she struggles to survive. She is preoccupied with basic needs (e.g.., food stamps) but her health conditions (e.g., memory loss and asthma) prevent her from following the requirements to access basic services that could improve her situation. Like Linda, several participants reported being unable to attend their PrEP appointments due to conflicts with appointments for mental health and substance use treatment, as well as not being able to remember things because they had so much going on. Ebony, 36-year-old cisgender Black woman who did not attend her PrEP appointment explained:

I'm too busy. I had to reset up my psych appointment and my therapist appointment and my ... I had to go see my other doc. I've been running around and going to my program [substance use program] five times a week, so I've just been a little busy.

Women in this study told study described juggling life circumstances, which included maintaining their own physical and mental health. Many women had chronic conditions that needed to be maintained and they felt that PrEP would not be 
feasible for them because of those commitments. Along with taking care of their own health, women also told us that they had others to care for, such as children or elderly parents and grandparents, which took up their time and would prevent them from attending PrEP appointments.

\section{Discussion}

Our qualitative study explored participants' perceptions of PrEP-UP, a novel PrEP peer outreach and navigation intervention for women based at mobile syringe exchange sites and sex worker and syringe exchange drop-in centers. Facilitators to PrEP-UP engagement were interest in PrEP, offering health and social services other than PrEP care, the women-focused approach, and peer outreach and navigation. Barriers to engaging in the PrEP-UP intervention were perceived HIV risk, concerns about medication side effects or interactions, housing insecurity and travel, co-occurring health-related conditions and appointments, and caregiving responsibilities.

Our study contributes to the very limited literature on PrEP interventions for cis and trans women. Recognizing that cis and trans women at risk for HIV face a number of competing health and social services needs due to the structural barriers they face, the intervention offered PrEP care referral along with referral to a number of other health and social services that women might need. We found that this aspect of the intervention was positively received by women, and many accepted the offer of non-PrEP health and social service referrals, however, not all women were able to access these services. This finding highlights the need to treat women holistically, acknowledging their varied life circumstances, many of which may be prioritized before PrEP. For example, women who are focused on finding a safe place to sleep at night or obtaining food for themselves and their families, may not have time to devote to PrEP appointments. Therefore, it is important to offer other needed resources along with PrEP in order to engage women throughout the PrEP care continuum [71, 72]. Importantly, our findings suggest that providing referrals to PrEP and other services are not enough. We recommend bundling PrEP care with other services in locations that are familiar and friendly to women who use drugs and/or exchange sex, such as syringe exchange programs (SEPs) or other community-based organizations, so that women can access numerous points of care in one place. For example, researchers in Philadelphia, PA have partnered with a local SEP and offered PrEP care onsite. They attribute the success of the PrEP intervention to it being offered at a local SEP where women were comfortable and already seeking care [73, 74]. In addition to embedding PrEP at familiar and often frequented locations, such as SEPs, providing same day PrEP is another important factor. Research has shown that same-day PrEP initiation is feasible and may engage more women throughout the PrEP care continuum. [75, 76].

There are few, if any, published women-focused PrEP interventions, so the finding that the women focus was favorable among our participants is a helpful insight for the design of future interventions to increase PrEP uptake among women. This approach may be particularly important as healthcare and drug treatment sites can be inhospitable to women, especially women who use drugs [77, 78]. Women are not always comfortable talking to their doctors about their HIV risk [79] and medical distrust is a barrier to PrEP [43]. Although our study did not unearth issues between cis and trans women past research has highlighted potential discomfort between the groups [80]. We likely did not find this in our study because we had a cisgender peer for cisgender women and a transgender peer for transgender women.

Along with the woman focus, participants noted they especially liked the peer aspect of PrEP-UP. Peer outreach and navigation have been successful tools to engage people in HIV care [47-49] and our study suggests peer navigation could be an important tool for future interventions aimed at engaging women in PrEP care.

Given that no women started PrEP, focusing on remedying the identified barriers to PrEP-UP will likely be critical for future interventions. Although past research with cisgender and transgender women has highlighted the need for gender affirming care, bundling referrals to other needed health and social services [80] along with the importance of peers [54], these are not sufficient; ultimately, women faced barriers that made attending PrEP appointments challenging. Barriers, such as competing priorities, travel, and other health-related conditions were significant [70]. The norm in healthcare of referring patients to services is a suboptimal approach to engaging women at risk. Indeed, Martinez et al. found that the average space people who injected drugs traveled daily in San Francisco, California was 1.5 miles [81]. Like New York, San Francisco has public transit, but still people did not move far. Engaging women in care at one location (e.g., one-stop shop location) where they are already are (in this case, for harm reduction services), rather than simply referring them to different locations, may be more beneficial, and could be a way to address the barriers that women described in this study. Research on HCV has found on-site testing and treatment at programs for substance use disorder to be effective [82-85]. On site testing and treatment for PrEP would directly address many of the travel and logistic barriers and the obligation of attending other health-related appointments and could mitigate other barriers. For example, if a trusting environment is established where women create community and are comfortable, and perhaps other services are offered, PrEP may be more 
successful. One potential space to embed PrEP is at SEPs [77, 86, 87]. In addition, the possibility of using mobile HIV testing units for PrEP provision should be explored. We also suggest offering childcare so women with children who do not have assistance with childcare can engage in PrEP care. Finally, given the prevalence of structural barriers, multilevel interventions to engage women in PrEP care would be beneficial $[72,88]$.

Despite its strengths, our study has several limitations. First, the PrEP-UP intervention was only offered in English which limited the inclusion of monolingual Spanishspeaking participants and other women who do not speak English. Second, we sampled women in NYC who actively engaged with a local CBO and therefore our findings may not be generalizable to other populations of women, including women in NYC who were not engaged with our partner CBO. Third, we were unable to sample a larger amount of trans women and we were only able to include the three trans women who participated in follow up interviews in this analysis. Given the small number of trans women we were unable to make meaningful comparisons between cis and trans women. It may be necessary to develop different interventions for cisgender versus transgender women. Creating a specific space for transgender women may be a culturally responsive way to foster community and comfort for the transgender women. Future studies should explore the differences in PrEP experiences between cisgender and transgender women. Fourth, data was collected prior to the COVID-19 pandemic. Prior to the pandemic, the New York City Department of Health and Mental Hygiene released a statement warning that persons who inject drugs are at risk for acquiring HIV due to structural, community, and behavioral factors [89], and there is reason to believe that the pandemic may exacerbate structural and community issues that place women at risk [90]. Therefore, future research should explore how the pandemic may have impacted HIV risk among cisgender and transgender women.

\section{Conclusion}

Our qualitative study identified barriers to and facilitators of a novel PrEP intervention for women at high risk for HIV infection. Women who were interested in PrEP-UP expressed an interest in learning about PrEP as well as other health and social services that we offered. Other facilitators include the intervention's women's focused approach as well as peer outreach and navigation component. Future interventions should also address identified barriers. Results from this study suggest that future PrEP interventions targeting women should be (1) offered in settings women already frequent and at which they are comfortable, (2) offer other services at that location that can holistically address women's needs, (3) utilize peers to recruit and educate women, (4) have a women's focus that incudes gender-affirming care, and (5) consider offering same-day PrEP.

Acknowledgements We are grateful to Tonia Poteat, $\mathrm{PhD}, \mathrm{PA}-\mathrm{C}$, $\mathrm{MPH}$, and Hilary Surratt, $\mathrm{PhD}$ for insightful discussions during the early stages of this project and to Axcel Barboza for his input into planning and implementation phases of this study. The authors would also like to thank colleagues at NDRI for their assistance with Peer training. This project was supported by a Centers for Disease Control and Prevention Minority HIV/AIDS Research Initiative Grant (1U01PS005111-01).

Funding This work was funded by the Centers for Disease Control and Prevention Minority HIV/AIDS Research Initiative Grant (1U01PS005111-01), PI: Oni J. Blackstock; T32 DA007233-31, PI: Falkin; and R25DA026401, PI Avelardo Valdez.

\section{References}

1. Kral AH, Bluthenthal RN, Lorvick J, Gee L, Bacchetti P, Edlin BR. Sexual transmission of HIV-1 among injection drug users in San Francisco, USA: risk-factor analysis. Lancet. 2001;357(9266):1397-401.

2. Rondinelli AJ, Ouellet LJ, Strathdee SA, Latka MH, Hudson SM, Hagan $\mathrm{H}$, et al. Young adult injection drug users in the United States continue to practice HIV risk behaviors. Drug Alcohol Depend. 2009;104(1-2):167-74.

3. Jenness SM, Kobrak P, Wendel T, Neaigus A, Murrill CS, Hagan $\mathrm{H}$. Patterns of exchange sex and HIV infection in high-risk heterosexual men and women. Save Reference. 2011;88(2):329-41.

4. Blouin K, Leclerc P, Morissette C, Roy E, Blanchette C, Parent $\mathrm{R}$, et al. Sex work as an emerging risk factor for human immunodeficiency virus seroconversion among people who inject drugs in the SurvUDI network. Sex Transm Dis. 2016;43(10):648-55.

5. Lemert EM. Social pathology: a systematic approach to the theory of sociopathic behavior. New York: McGraw-Hill; 1951.

6. Herbst JH, Jacobs ED, Finlayson TJ, McKleroy VS, Neumann MS, Crepaz N, et al. Estimating HIV prevalence and risk behaviors of transgender persons in the United States: a systematic review. AIDS Behav. 2008;12(1):1-17.

7. Walters SM, Reilly KH, Neaigus A, Braunstein S. Awareness of pre-exposure prophylaxis (PrEP) among women who inject drugs in NYC: the importance of networks and syringe exchange programs for HIV prevention. Harm Reduct J. 2017;14(1):40.

8. Peters PJ, Pontones P, Hoover KW, Patel MR, Galang RR, Shields $\mathrm{J}$, et al. HIV infection linked to injection use of oxymorphone in Indiana, 2014-2015. N Engl J Med. 2016;375(3):229-39.

9. Cranston K, Alpren C, John B, Dawson E, Roosevelt K, Burrage A, et al. Notes from the field: HIV diagnoses among persons who inject drugs-Northeastern Massachusetts, 2015-2018. Morb Mortal Wkly Rep. 2019;68(10):253.

10. Golden MR, Lechtenberg R, Glick SN, Dombrowski J, Duchin J, Reuer JR, et al. Outbreak of human immunodeficiency virus infection among heterosexual persons who are living homeless and inject drugs-Seattle, Washington, 2018. Morb Mortal Wkly Rep. 2019;68(15):344.

11. Walters SM, Rivera AV, Reilly KH, Anderson BJ, Bolden B, Wogayehu A, et al. Exchange sex among persons who inject drugs 
in the New York metropolitan area: the importance of local context, gender and sexual identity. AIDS Behav. 2018;1:1-15.

12. Kalichman SC, Williams EA, Cherry C, Belcher L, Nachimson D. Sexual coercion, domestic violence, and negotiating condom use among low-income African American women. J Womens Health. 1998;7(3):371-8.

13. Pyett PM, Warr DJ. Vulnerability on the streets: female sex workers and HIV risk. AIDS Care. 1997;9(5):539-47.

14. Nemoto T, Operario D, Keatley J, Villegas D. Social context of HIV risk behaviours among male-to-female transgenders of colour. AIDS Care. 2004;16(6):724-35.

15. Pulerwitz J, Amaro H, Jong WD, Gortmaker SL, Rudd R. Relationship power, condom use and HIV risk among women in the USA. AIDS Care. 2002;14(6):789-800.

16. Bennett GA, Velleman RD, Barter G, Bradbury C. Gender differences in sharing injecting equipment by drug users in England. AIDS Care. 2000;12(1):77-87.

17. Evans JL, Hahn JA, Page-Shafer K, Lum PJ, Stein ES, Davidson PJ, et al. Gender differences in sexual and injection risk behavior among active young injection drug users in San Francisco (the UFO Study). J Urban Health. 2003;80(1):137-46.

18. Kurtz SP, Surratt HL, Kiley MC, Inciardi JA. Barriers to health and social services for street-based sex workers. J Health Care Poor Underserv. 2005;16(2):345-61.

19. Jaiswal J, Dunlap K, Griffin M, Cox A, Singer S, Hascher K, et al. Pre-exposure prophylaxis awareness, acceptability and potential stigma among medical and non-medical clinic staff in methadone treatment settings in northern New Jersey: the key role of nonmedical staff in enhancing HIV prevention. J Substance Abuse Treat. 2021;129:108371.

20. Baeten JM, Donnell D, Ndase P, Mugo NR, Campbell JD, Wangisi $\mathrm{J}$, et al. Antiretroviral prophylaxis for HIV prevention in heterosexual men and women. N Engl J Med. 2012;367(5):399-410.

21. Grant RM, Lama JR, Anderson PL, McMahan V, Liu AY, Vargas L, et al. Preexposure chemoprophylaxis for HIV prevention in men who have sex with men. N Engl J Med. 2010;363(27):2587-99.

22. Thigpen MC, Kebaabetswe PM, Paxton LA, Smith DK, Rose CE, Segolodi TM, et al. Antiretroviral preexposure prophylaxis for heterosexual HIV transmission in Botswana. N Engl J Med. 2012;367(5):423-34.

23. Deutsch MB, Glidden DV, Sevelius J, Keatley J, McMahan V, Guanira J, et al. HIV pre-exposure prophylaxis in transgender women: a subgroup analysis of the iPrEx trial. Lancet HIV. 2015;2(12):e512-9.

24. Thomson KA, Baeten JM, Mugo NR, Bekker L-G, Celum CL, Heffron R. Tenofovir-based oral preexposure prophylaxis prevents HIV infection among women. Curr Opin HIV AIDS. 2016;11(1):18-26.

25. Tomko C, Park JN, Allen ST, Glick J, Galai N, Decker MR, et al. Awareness and interest in HIV pre-exposure prophylaxis among street-based female sex workers: results from a US context. AIDS Patient Care STDs. 2019;33:49-57.

26. Neaigus A, Reilly KH, Jenness SM, Hagan H, Wendel T, GelpiAcosta C. Dual HIV risk: receptive syringe sharing and unprotected sex among HIV-negative injection drug users in New York City. AIDS Behav. 2013;17(7):2501-9.

27. Blackstock OJ, Patel VV, Felsen U, Park C, Jain S. Pre-exposure prophylaxis prescribing and retention in care among heterosexual women at a community-based comprehensive sexual health clinic. AIDS Care. 2017;29(7):866-9.

28. Seidman DL, Weber S, Timoney MT, Oza KK, et al. Use of HIV pre-exposure prophylaxis during the preconception, antepartum and postpartum periods at two United States medical centers. AIDS Care. 2016;215(5):632.

29. Hodges-Mameletzis I, Fonner VA, Dalal S, Mugo N, Msimanga-Radebe B, Baggaley R. Pre-exposure prophylaxis for hiv prevention in women: current status and future directions. Drugs. 2019;79(12):1263-76.

30. Walters SM, Rivera AV, Starbuck L, Reilly KH, Boldon N, Anderson BJ, et al. Differences in awareness of pre-exposure prophylaxis and post-exposure prophylaxis among groups at-risk for HIV in New York State: New York City and Long Island, NY, 2011-2013. JAIDS. 2017;75:S383-91.

31. Walters SM, Kral AH, Simpson KA, Wenger L, Bluthenthal RN. HIV pre-exposure prophylaxis prevention awareness, willingness, and perceived barriers among people who inject drugs in Los Angeles and San Francisco, CA 2016-2018. Substance Use Misuse. 2020;1:1-11.

32. Kuhns LM, Reisner SL, Mimiaga MJ, Gayles T, Shelendich M, Garofalo R. Correlates of PrEP indication in a multi-site cohort of young HIV-uninfected transgender women. AIDS Behav. 2015;20:1470-7.

33. Wilson EC, Jin H, Liu A, Raymond HF. Knowledge, indications and willingness to take pre-exposure prophylaxis among transwomen in San Francisco, 2013. PLoS ONE. 2015;10(6):e0128971.

34. Bazzi AR, Biancarelli DL, Childs E, Drainoni M-L, Edeza A, Salhaney $\mathrm{P}$, et al. Limited knowledge and mixed interest in preexposure prophylaxis for HIV prevention among people who inject drugs. AIDS Patient Care STDs. 2018;32:529-37.

35. Park CJ, Taylor TN, Gutierrez NR, Zingman BS, Blackstock OJ. Pathways to HIV pre-exposure prophylaxis among women prescribed PrEP at an urban sexual health clinic. J Assoc Nurs AIDS Care. 2019;30:321-9.

36. Nunn A, Zaller N, Cornwall A, Mayer KH, Moore E, Dickman S, et al. Low perceived risk and high HIV prevalence among a predominantly African American population participating in Philadelphia's rapid HIV testing program. AIDS Patient Care STDS. 2011;25(4):229-35.

37. Auerbach JD, Kinsky S, Brown G, Charles V. Knowledge, attitudes, and likelihood of pre-exposure prophylaxis (PrEP) use among US women at risk of acquiring HIV. AIDS Patient Care STDS. 2015;29(2):102-10.

38. Flash CA, Stone VE, Mitty JA, Mimiaga MJ, Hall KT, Krakower $\mathrm{D}$, et al. Perspectives on HIV prevention among urban Black women: a potential role for HIV pre-exposure prophylaxis. AIDS Patient Care STDS. 2014;28(12):635-42.

39. Rael CT, Martinez M, Giguere R, Bockting W, Maccrate C, Mellman W, et al. Barriers and facilitators to oral PrEP use among transgender women in New York City. AIDS Behav. 2018;22:3627-36.

40. Park C, Taylor T, Rios N, Khedimi R, Weiss C, Dolce E, et al editors. Perspectives of women prescribed HIV pre-exposure prophylaxis (PrEP). Open forum infectious diseases. Oxford: Oxford University Press; 2017.

41. Braksmajer A, Senn TE, McMahon J. the potential of pre-exposure prophylaxis for women in violent relationships. AIDS Patient Care STDS. 2016;30(6):274-81.

42. Sevelius JM, Keatley J, Calma N, Arnold E. 'I am not a man': trans-specific barriers and facilitators to PrEP acceptability among transgender women. Glob Public Health. 2016;11(7-8):1060-75.

43. Bradley E, Forsberg K, Betts JE, DeLuca JB, Kamitami E, Porter $\mathrm{SE}$, et al. Factors affecting pre-exposure prophylaxis implementation for women in the United States: A systematic review. J Women's Health. 2019;28:1272-85.

44. Aaron E, Blum C, Seidman D, Hoyt MJ, Simone J, Sullivan $\mathrm{M}$, et al. Optimizing delivery of hiv preexposure prophylaxis for women in the United States. AIDS Patient Care STDS. 2018;32(1):16-23.

45. Johnson LM, Green HD Jr, Koch B, Stockman JK, Felsher M, Roth AM, et al. Role of social networks and social norms in future PrEP use in a racially diverse sample of at-risk women and members of their social networks. JAIDS. 2021;86(4):422-9. 
46. Glick JL, Russo R, Jivapong B, Rosman L, Pelaez D, Footer KHA, et al. The PrEP care continuum among cisgender women who sell sex and/or use drugs globally: a systematic review. AIDS Behav. 2020;24(5):1312-33.

47. Bradford JB, Coleman S, Cunningham W. HIV system navigation: an emerging model to improve HIV care access. AIDS Behav. 2007;21(s1):S49-58.

48. Higa DH, Marks G, Crepaz N, Liau A, Lyles CM. Interventions to improve retention in HIV primary care: a systematic review of US studies. Curr HIV/AIDS Rep. 2012;9(4):313-25.

49. Rajabiun $\mathrm{S}, \mathrm{Cabral} H$, Tobias $\mathrm{C}$, Relf M. Program design and evaluation strategies for the special projects of national significance outreach initiative. AIDS Patient Care STDs. 2007;21(s1):S9-19.

50. Davidson L, Amy CB, Guy K, Er RAM. Peer support among persons with severe mental illnesses: a review of evidence and experience. World Psychiatry. 2012;11(2):123-8.

51. Sledge WH, Lawless M, Sells D, Wieland M, O'Connell MJ, Davidson L. Effectiveness of peer support in reducing readmissions of persons with multiple psychiatric hospitalizations. Psychiatr Serv. 2011;62(5):541-4.

52. Bassuk EL, Hanson J, Greene RN, Richard M, Laudet A. Peerdelivered recovery support services for addictions in the United States: a systematic review. J Subst Abuse Treat. 2016;63:1-9.

53. Bardwell G, Kerr T, Boyd J, McNeil R. Characterizing peer roles in an overdose crisis: preferences for peer workers in overdose response programs in emergency shelters. Drug Alcohol Depend. 2018;190:6-8.

54. Lennox R, Lamarche L, O'Shea T. Peer support workers as a bridge: a qualitative study exploring the role of peer support workers in the care of people who use drugs during and after hospitalization. Harm Reduct J. 2021;18(1):467.

55. Blackstock OJ, Platt J, Golub SA, Anakaraonye AR, Norton BL, Walters SM, et al. A pilot study to evaluate a novel pre-exposure prophylaxis peer outreach and navigation intervention for women at high risk for HIV infection. AIDS Behav. 2020;25:1411-22.

56. HIV Surveillance Annual Report, 2018 (Rep.). (2019, November). Retrieved May 07, 2020, from New York City Department of Health and Mental Hygiene website: https://www1.nyc.gov/assets/ doh/downloads/pdf/dires/hiv-surveillance-annualreport-2018.pdf

57. DiClemente RJ, Crosby RA, Kegler MC. Emerging theories in health promotion practice and research. Hoboken: Wiley; 2009.

58. Grant RM, Pellegrini M, Defechereux PA, Anderson PL, Yu M, Glidden DV, et al. Sex hormone therapy and tenofovir diphosphate concentration in dried blood spots: primary results of the iBrEATHe study. Clin Infect Dis. 2020. https://doi.org/10.1093/ $\mathrm{cid} / \mathrm{ciaa} 1160$.

59. Lee R, Cui RR, Muessig KE, Thirumurthy H, Tucker JDJA. Incentivizing HIV/STI testing: a systematic review of the literature. Behavior. 2014;18(5):905-12.

60. Stopka TJ, Marshall C, Bluthenthal RN, Webb DS, Truax SR. $\mathrm{HCV}$ and HIV counseling and testing integration in California: an innovative approach to increase HIV counseling and testing rates. Public Health Rep. 2007;122(2 suppl):68-73.

61. Abadie R, Brown B, Fisher CB. "Money Helps": people who inject drugs and their perceptions of financial compensation and its ethical implications. Ethics Behav. 2018;29:607-29.

62. Charmaz K. Constructing grounded theory. Thousand Oaks: Sage; 2014.

63. Braun V, Clarke V. Thematic analysis. Washington: APA; 2012.

64. MacQueen KM, McLellan E, Kay K, Milstein B. Codebook development for team-based qualitative analysis. CAM J. 1998;10(2):31-6.

65. Miles MB, Huberman AM, Saldana J. Qualitative data analysis. Tbousand Oaks: Sage; 2013
66. Orne J, Bell M. An invitation to qualitative fieldwork: a multilogical approach. New York: Routledge; 2015.

67. Aidala A, Cross JE, Stall R, Harre D, Sumartojo E. Housing status and HIV risk behaviors: implications for prevention and policy. AIDS Behav. 2005;9(3):251-65.

68. Stoner MCD, Haley DF, Golin CE, Adimora AA, Pettifor A. The relationship between economic deprivation, housing instability and transactional sex among women in North Carolina (HPTN 064). AIDS Behav. 2019;23:2946-55.

69. Dunkle KL, Wingood GM, Camp CM, DiClemente RJ. Economically motivated relationships and transactional sex among unmarried African American and white women: Results from a US national telephone survey. Public Health Rep. 2010;125:90-100.

70. Qin Y, Price C, Rutledge R, Puglisi L, Madden LM, Meyer JP. Women's decision-making about PrEP for HIV prevention in drug treatment contexts. JIAPAC. 2020;19:232595821990009.

71. Wilson EC, Turner CM, Arayasirikul S, Lightfoot M, Scheer S, Raymond HF, et al. Disparities in the PrEP continuum for trans women compared to MSM in San Francisco, California: Results from population-based cross-sectional behavioural surveillance studies. J Int AIDS Soc. 2020;23:S3.

72. Nydegger LA, Dickson-Gomez J, Ko TK. Structural and syndemic barriers to PrEP adoption among Black women at high risk for HIV: a qualitative exploration. Cult Health Sex. 2020;23:659-73.

73. Felsher M, Ziegler E, Smith LR, Sherman SG, Amico KR, Fox $\mathrm{R}$, et al. An Exploration of pre-exposure prophylaxis (PrEP) initiation among women who inject drugs. Arch Sex Behav. 2020;49:2205-10.

74. Felsher M, Piecara B, Krakower D, Bellamy S, Laurano R, Van Der Pol B, et al., editors. Offering PrEP Care within a Community-based Syringe Exchange Facilitates Uptake among Women Who Inject Drugs. APHA's 2019 Annual Meeting and Expo (Nov 2-Nov 6); 2019: American Public Health Association.

75. Kamis KF, Marx GE, Scott KA, Gardner EM, Wendel KA, Scott ML, et al. Same-day HIV pre-exposure prophylaxis (PrEP) initiation during drop-in sexually transmitted diseases clinic appointments is a highly acceptable, feasible, and safe model that engages individuals at risk for HIV into PrEP care. Open Forum Infect Dis. 2019;6:7.

76. Rowan SE, Patel RR, Schneider JA, Smith DK. Same-day prescribing of daily oral pre-exposure prophylaxis for HIV prevention. Lancet HIV. 2020;8:e114-20.

77. Muncan B, Walters SM, Ezell J, Ompad DC. "They look at us like junkies": influences of drug use stigma on the healthcare engagement of people who inject drugs in New York City. Harm Reduct J. 2020;17:1

78. Earnshaw V, Smith L, Copenhaver M. Drug addiction stigma in the context of methadone maintenance therapy: an investigation into understudied sources of stigma. Int J Ment Health Addict. 2013;11(1):110-22.

79. Goparaju L, Praschan NC, Jeanpiere LW, Experton LS, Young MA, Kassaye S. Stigma, partners, providers and costs: Potential barriers to PrEP uptake among US women. J AIDS Clin Res. 2017;08:09.

80. Auerbach JD, Moran L, Watson C, Weber S, Keatley J, Sevelius $\mathrm{J}$. We are all women: barriers and facilitators to inclusion of transgender women in HIV treatment and support services designed for cisgender women. AIDS Patient Care STDS. 2020;34(9):392-8.

81. Martinez AN, Lorvick J, Kral AH. Activity spaces among injection drug users in San Francisco. Int J Drug Policy. 2014;25(3):516-24.

82. Butner JL, Gupta N, Fabian C, Henry S, Shi JM, Tetrault $\mathrm{JM}$. Onsite treatment of $\mathrm{HCV}$ infection with direct acting 
antivirals within an opioid treatment program. J Subst Abuse Treat. 2017;75:49-53.

83. Carey KJ, Huang W, Linas BP, Tsui JI. Hepatitis C virus testing and treatment among persons receiving buprenorphine in an office-based program for opioid use disorders. J Subst Abuse Treat. 2016;66:54-9.

84. Masson CL, Delucchi KL, McKnight C, Hettema J, Khalili M, Min A, et al. A randomized trial of a hepatitis care coordination model in methadone maintenance treatment. Am J Public Health. 2013;103(10):e81-8.

85. Muncan B, Jordan AE, Perlman DC, Frank D, Ompad DC, Walters SM. Acceptability and effectiveness of hepatitis $C$ care at syringe service programs for people who inject drugs in New York city. Subst Use Misuse. 2021;1:1-10.

86. Walters SM, Coston B, Neaigus A, Rivera AV, Starbuck L, Ramirez $\mathrm{V}$, et al. The role of syringe exchange programs and sexual identity in awareness of pre-exposure prophylaxis (PrEP) for male persons who inject drugs. Int J Drug Policy. 2020;77:102671.

87. Roth AM, Aumaier BL, Felsher MA, Welles SL, MartinezDonate AP, Chavis M, et al. An exploration of factors impacting preexposure prophylaxis eligibility and access among syringe exchange users. Sex Transm Dis. 2018;45(4):217-21.

88. Pinto RM, Berringer KR, Melendez R, Mmeje O. Improving PrEP implementation through multilevel interventions: a synthesis of the literature. AIDS Behav. 2018;22(11):3681-91.

89. New York City Department of Health and Mental Hygiene. Risk factors that contributed to HIV outbreaks in Massachusetts are present in New York City. 2019 Advisory (No. 3). https://www1. nyc.gov/assets/doh/downloads/pdf/han/advisory/2019/risk-facto rs-HIV-outbreaks-present-in-NYC.pdf. Published 2019. Accessed 7 May 2020.

90. Walters S. COVID-19 and people who use drugs: a commentary. Health Behav Policy Rev. 2020;7(5):489-97.

Publisher's Note Springer Nature remains neutral with regard to jurisdictional claims in published maps and institutional affiliations. 